\title{
UNA CARTA DE KENNETH SISAM A RONALD SYME Y SU RESPUESTA: JUNIO DE 1939
}

\author{
Gustavo A. Vivas García ${ }^{1}$
}

\section{Resumen}

El archivo editorial de The Roman Revolution puede consultarse en la sede de la Oxford University Press. Ahí se encuentran dos interesantes documentos fechados a mediados de junio de 1939, aproximadamente dos meses y medio antes de la publicación de la famosa monografía escrita por Syme. Se trata de una carta mecanografiada de Kenneth Sisam, alto cargo de la editorial, dirigida a Ronald Syme y fechada el martes 13 de junio de 1939 y la respuesta de éste, autógrafa y enviada al día siguiente miércoles 14 de junio, desde el Trinity College. Este trabajo analiza de manera pormenorizada y por extenso estas dos cartas.

\section{Palabras Clave}

Ronald Syme; Kenneth Sisam; The Roman Revolution; Oxford.

\section{Resumo}

O arquivo editorial de The Roman Revolution pode ser consultado na sede da Oxford University Press. Lá se encontram interesantes documentos datados de meados de junho de 1939, aproximadamente dois meses e meio antes da publicação da famosa monografia escrita por Syme. Trata-se de uma carta datilografada de keneth Sisam, alto funcionario da editora, dirigida a Ronald Syme e datada de terça-feira, 13 de junho de 1939 e a resposta desta, assinada e enviada no dia seguinte, quarta-feira, 14 de junho, a partir do Trinity College. Este trabalho analisa em detalhe extensivamente estas duas cartas.

\section{Palavras-chave}

Ronald Syme; Kenneth Sisam; The Roman Revolution; Oxford.

\footnotetext{
${ }^{1}$ Profesor Doctor - Universidad de La Laguna, San Cristóbal de La Laguna, España. E-mail: gusgarvi@gmail.com
} 
Dentro del archivo editorial de The Roman Revolution [en adelante $R R$ ] que, con el título "Augustus: Leader and Party", puede consultarse en la sede de la Oxford University Press - OUP - (Vivas García, 2017: 284s. y n.31) $)^{2}$; se encuentran dos interesantes documentos fechados a mediados de junio de 1939, aproximadamente dos meses y medio antes de la publicación de la que se convertirá, a la postre, en la obra cumbre de Ronald Syme, entonces a la sazón un investigador de 36 años de edad. Hablamos, por supuesto, de su conocida obra "La Revolución Romana" cuya primera edición inglesa vió la luz el 7 de septiembre de ese año (Vivas García, 2016: 170s.).

Se trata de una carta mecanografiada escrita, seguramente en Oxford, por Kenneth Sisam a Ronald Syme y fechada el martes 13 de junio de 1939 y la respuesta de éste, autógrafa y enviada desde el Trinity College donde profesaba desde hacía ya una década, el día siguiente miércoles 14 de junio.

Kenneth Sisam (1887-1971), medievalista en Oxford de origen neozelandés como Syme, con el que compartía "patria chica" y gran experto en literatura medieval inglesa, fue tutor y amigo personal de J.R.R. Tolkien y alto cargo de la OUP, en la que comenzaría a trabajar en 1915. Fue Secretario Asistente de la editorial durante veinte años de 1922 a 1942 y culminó su carrera como ejecutivo de la misma, en calidad de Secretario de los Delegados, desde 1942 a 1948. Sisam fue amigo personal de Syme y era la "eminencia gris" que estuvo detrás, casi con toda seguridad, de la composición y publicación de $R R$ $(\text { Stray, online })^{3}$. A él se debe también, con bastante probabilidad, la sugerencia o decisión del cambio de título que desde la órbita de la editorial se le hizo a Syme para que modificara el nombre de su monografía, meses antes de su publicación, de "Augustus: Leader and Party" a "The Roman Revolution" (vd. Vivas García, 2017: ibid. y 2018: en prensa). La carta de Sisam se reproduce a continuación:

\footnotetext{
2 Archivo editorial BACKB 00551. Agradezco al Prof. Dr. Anthony R. Birley la generosa ayuda prestada durante la composición de este trabajo. Y también, como viene siendo habitual desde hace años, sus consejos siempre oportunos.

${ }^{3}$ El Prof. Christopher Stray me proporcionó una copia de su trabajo de manera inmediata y generosa.
} 


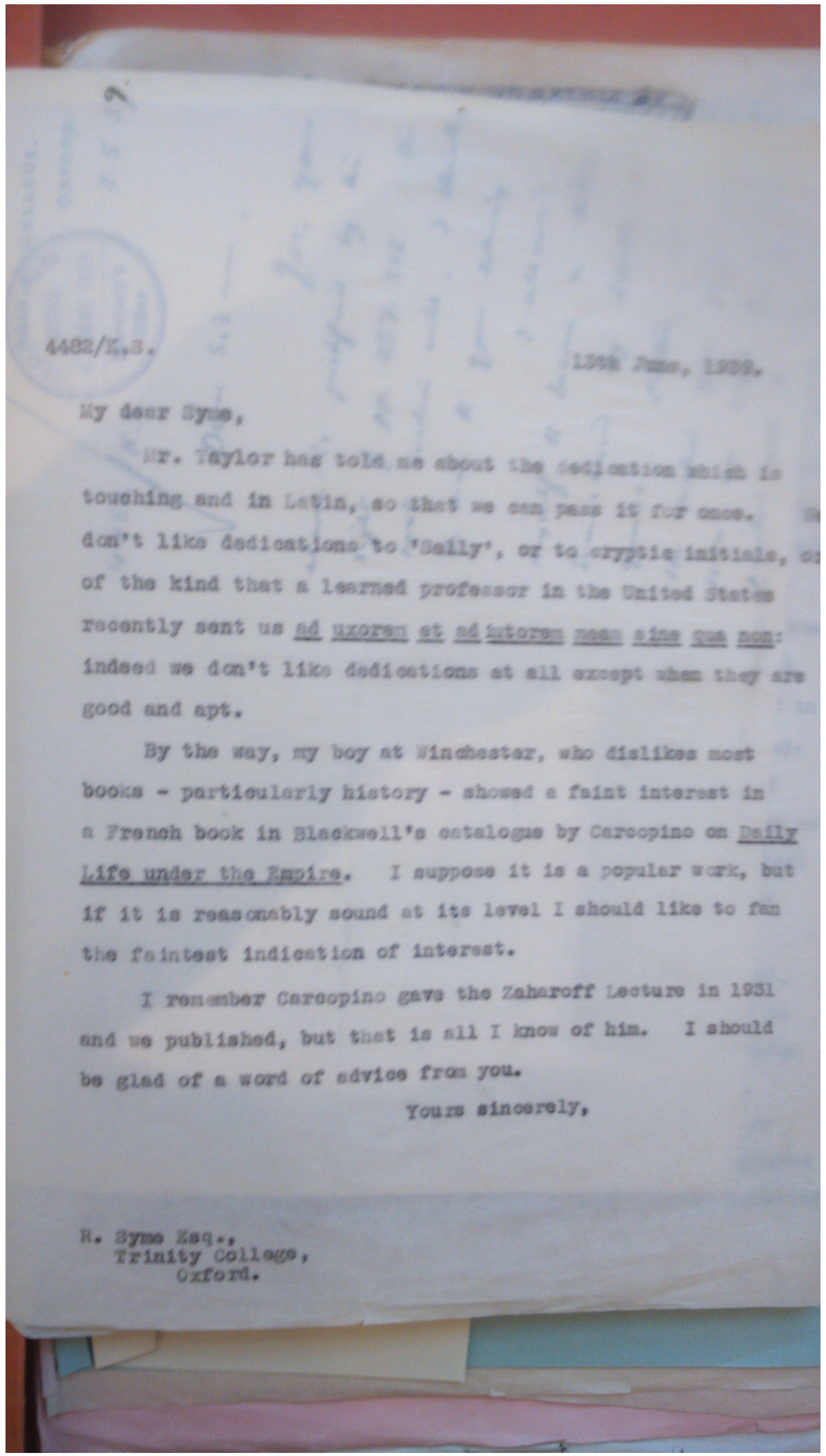

Heródoto, Unifesp, Guarulhos, v. 3, n. 2, Dezembro, 2018. p. 277-287 
La transcripción de la misiva es la siguiente:

“4482/ K.S.

13th June, 1939.

My dear Syme,

Mr. Taylor has told me about the dedication which is / touching and in Latin, so that we can pass it for once. We/ don't like dedications to 'Sally', or to cryptic initials, or/ of the kind that a learned professor in the United States/ recently sent us ad uxorem et ad iutorem meam sine qua non:/ indeed we don't like dedications at all except when they are/good and apt.

By the way, my boy at Winchester, who dislikes most/ books - particularly history - showed a faint interest in/ a French book in Blackwell's catalogue by Carcopino on Daily/Life under the Empire. I suppose it is a popular work, but/ if it is reasonably sound at its level I should like to fan/ the faintest indication of interest./

I remember Carcopino gave the Zaharoff Lecture in 1931/ and we published, but that is all I know from him. I should/ be glad of a word of advice from you./

Yours sincerely,/

R. Syme Esq.,

Trinity College,

Oxford."

La carta de Sisam comienza indicando que un desconocido señor Taylor, seguramente un empleado de la editorial quizá encargado de labores de secretariado o colaborador de Sisam, le ha comunicado la dedicatoria que Syme había decidido colocar en el frontispicio de su obra: se trata de la frase latina PARENTIBVS OPTIMIS PATRIAEQVE $(R R, \mathrm{v})$. 
La dedicatoria es una oración sintética muy del gusto de Syme, que posee un punto nostálgico, y podemos traducir como "A mis padres y a mi patria". La elección del citado enunciado pone de relieve algunos detalles. Esta ocasión fue la primera y también la última vez que Syme dedicó uno de sus libros. Jamás volvería a hacerlo, a ninguna otra persona ni institución. La mención a sus progenitores puede no sorprendernos, pero $¿ y$ la de su patria, obviamente referida a su isla natal de Nueva Zelanda?

Syme se sintió durante toda su vida un "provincial" que profesaba en uno de los santuarios del saber de la metrópoli. No en vano, una de las claves investigadoras de la producción symeana era el análisis de la condición del "provincial" en la antigua Roma. Además de la situación del extranjero en provincias y, no lo olvidemos, su tratamiento y análisis de personajes como Tácito, Ovidio o Dión, todos ellos hombres de condición periférica incardinados en la Urbs (Bowersock, 1994: 539-563).

Syme llegó a Oxford desde las antípodas, donde nació y vivió hasta 1925. A pesar de desarrollar el resto de su carrera profesional en Oxford, con el paréntesis de su servicio en el Gobierno de Su Majestad durante la segunda de las conflagraciones mundiales, siempre sintió que Nueva Zelanda era su auténtica y verdadera patria. De hecho, mantuvo la ciudadanía neozelandesa y el pasaporte de ese país hasta su muerte, lo que le convertiría en protagonista de alguna que otra anécdota, entre chocante y divertida, a su paso por los controles de pasaporte de alguno de los numerosos países que visitó durante su vida. Ese especial cariño a su "patria chica" explica la dedicatoria, que Sisam califica de "touching", junto con el recuerdo a sus padres.

Sisam señala que en la centenaria editorial no agradaban las dedicatorias en los libros que publicaban, en general, y refiere a renglón seguido una escrita por un catedrático estadounidense, del que no da más datos, y que reza: "ad uxorem et ad iutorem meam sine qua non", es decir, " a mi esposa y ayudante sin la que no hubiera sido posible" [la composición del libro o monografía dedicado, se entiende].

Estamos ante una dedicatoria inusual en su formulación, y esa es quizá la razón por la que Sisam la menciona en esta carta. El término iutor no existe en latín, el vocablo es adiutor, nombre de agente del verbo adiuvo, que significa 'ayudar', y ése es el sentido del texto. La única explicación que puede existir para ad iutorem es que el autor de la dedicatoria haya entendido un sintagma nominal con la preposición ad, olvidando que ya el verbo adiuvo lleva el preverbio que precisa, por tanto, el significado del verbo simple $e^{4}$.

\footnotetext{
${ }_{4}^{4}$ Agradezco la ayuda decisiva prestada por la Prof. María del Pilar Lojendio, colega del Área de Filología Clásica de la ULL, en lo que se refiere a esta cuestión tan específica.
} 
Puede esgrimirse otra explicación, más sencilla. Sisam debió escribir sin duda adiutorem y no ad iutorem. Pero la carta fue mecanografiada por un colaborador, que entendió la segunda partícula "ad" de la misma manera que la primera y ahí radica la equivocación. Todo parece ser un error de mecanografiado de un secretario o secretaria, que Sisam no se preocupó en corregir o que simplemente no tuvo en cuenta.

Sabemos, por el segundo párrafo de la carta, que el hijo de Sisam estudiaba en ese momento en Winchester College, una escuela privada masculina fundada en 1382 por William de Wykeham, obispo de Winchester, y canciller de Eduardo III y Ricardo II. El colegio se ubica en la ciudad inglesa homónima, antigua capital sajona de Inglaterra.

Winchester College sigue en funcionamiento hoy en día tras más de seiscientos años de historia ininterrumpida, siendo el único college con dicha característica. Es, y lo era también en 1939, un colegio de carácter elitista, de difícil acceso y muy caro, ejemplo acrisolado de la tradición educativa que durante siglos ha caracterizado a la enseñanza privada de Gran Bretaña. De hecho, Winchester sirvió de modelo para la fundación, medio siglo más tarde, de Eton y de King's College en Cambridge.

El hijo de Sisam al que sabemos, por su padre, que no le gustaba la lectura en general, y mucho menos los libros de temática histórica, pareció demostrar un débil interés por un libro escrito en francés del historiador Jérôme Carcopino (1881 - 1970). El libro en cuestión es "La Vie quotidienne à Rome à l'apogée de l'Empire", editado en París por Hachette en una primera edición en 1936. Hubo otra edición en 1939. No sabemos cuál de las dos es la que causó el interés del hijo del editor, al verla en el catálogo de la famosa librería oxoniense Blackwell, que desde 1879 hasta la actualidad se erige en "the Broad" como faro bibliófilo en su establecimiento original de la ciudad universitaria británica ${ }^{5}$.

Sisam escribe, casi como conclusión de su carta en el último párrafo, que "Carcopino gave the Zaharoff Lecture in 1931 and we published". En efecto, la conferencia del historiador francés titulada "Ce que Rome et l'Empire Romain doivent à la Gaule" fue publicada por la Clarendon Press en un libreto de 36 páginas en 1932, siendo reseñada muy brevemente de forma positiva por el profesor A. N. Newell de la universidad de Manchester en el número de 1932 de la revista The Classical Review.

\footnotetext{
${ }^{5}$ Quizá procede recordar aquí la oda a esta excelente librería compuesta por el poeta inglés John Masefield (1878-1967), Poeta Laureado, que comienza: "I seek few treasures, except books, the tools/ Of those celestial souls the world calls fools./ Happy the morning giving time to stop/ An hour at once in Basil Blackwell's shop/ There, in the Broad, within whose booky house/ Half of England's scholars nibble books or browse/...".
} 
Por cierto la Conferencia Zaharoff, a la que se invitó a Carcopino, fue establecida en 1918 después de una donación de 25.000 libras esterlinas que el industrial, traficante de armas y filántropo Sir Basil Zaharoff (1839-1936) concedió al Departamento de Francés de la universidad para, entre otras disposiciones, invitar a eminentes figuras de la intelligentsia cultural francesa a Oxford a pronunciar lecciones magistrales y conferencias.

La respuesta de Syme no se hizo esperar y está fechada el día siguiente, 14 de junio en un papel con membrete del Trinity College. La reproducimos a continuación:

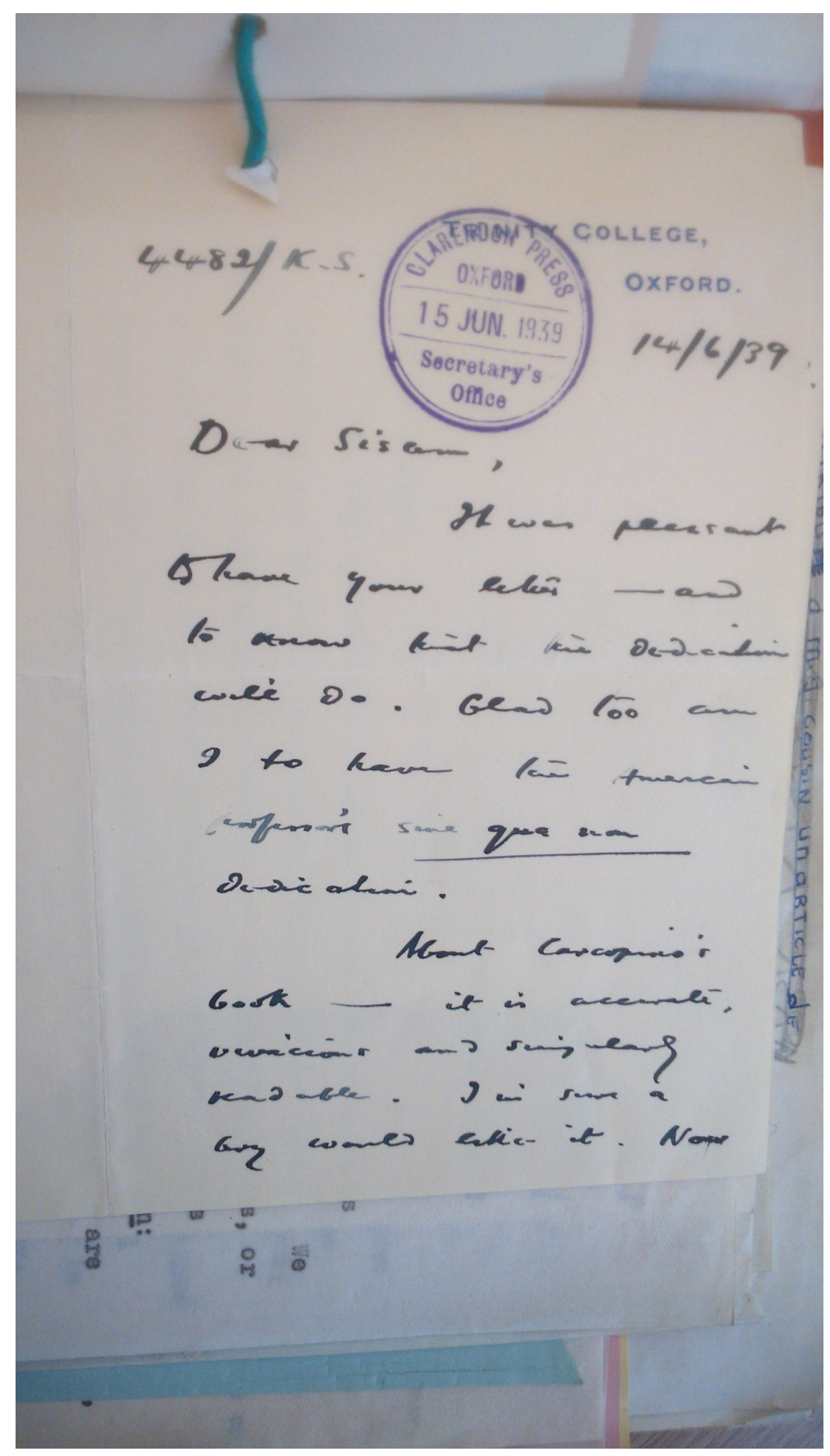




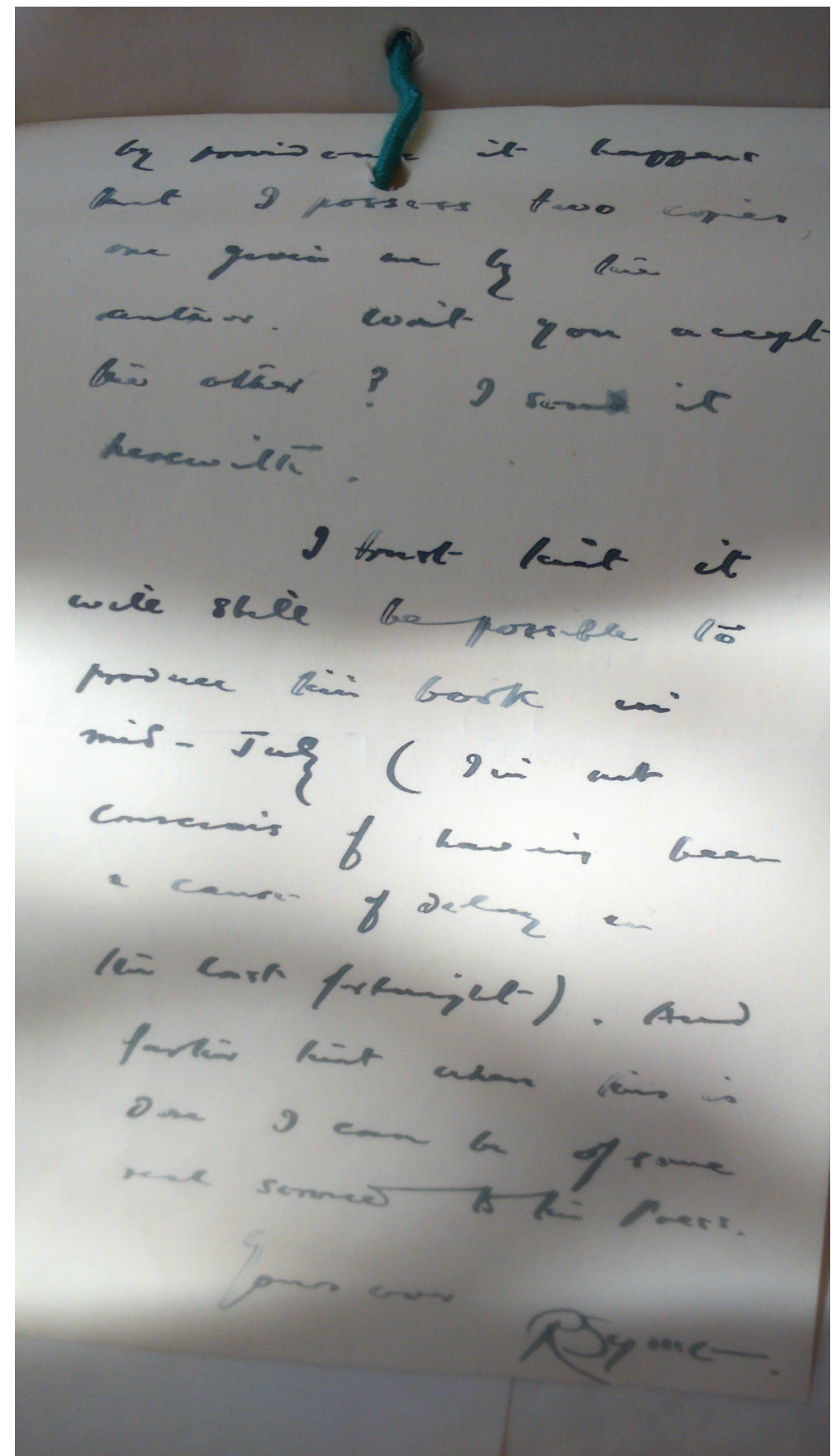

La transcripción de la carta es como sigue:

$$
\begin{aligned}
& \text { “4482/ K.S. } \\
& \text { 14/06/39 }
\end{aligned}
$$

\section{Dear Sisam,/}

It was pleasant/ to have your letter -and/ to know that the dedication/ will do. Glad too am/ I to have the American/ professor's sine qua non / dedication. / 
About Carcopino's / book -it is accurate,/ vivacious and singularly/ readable. I am sure a/ boy would like it. Now/ by providence it happens/ that I possess two copies/ one given me by the/ author. Won't you accept/ the other? I send it/ herewith.

I trust that it / will still be possible to/ produce the book [i.e. RR] in/ mid-July (I'm not/ conscious of having been / a cause of delay in / the last fortnight). And / further that when this is/ done I can be of some / real service to the Press.

\section{Yours ever/}

RSyme"

Como vemos, en la respuesta a la misiva de su compatriota editor, Syme se muestra contento de recibir la dedicatoria del profesor estadounidense. La mención de Sisam a su hijo en su carta y el hecho de que Syme en su respuesta le enviara un ejemplar del libro de Carcopino para su vástago, indica que seguramente ambos mantenían una muy buena relación.

En un primer momento, el lanzamiento de $R R$ estaba previsto para julio, de ahí la mención de Syme en su carta, a pesar de que la monografía se publicó finalmente el 7 de septiembre. No sabemos exactamente que causó ese retraso de casi dos meses, pero podemos especular sobre las razones y ahí estriba la importancia de esta carta de Ronald Syme.

Su manuscrito de casi 800 páginas (vd. Vivas García, 2018: en prensa), debe haber sido difícil de editar, por lo largo y complejo del mismo en lo referente a su mecanografiado y maquetado. Es factible que hiciera una gran cantidad de cambios en las galeradas finales. Quizá también ayude a comprender el retraso de Syme, al que se refiere en esta carta durante "the last fornight", el hecho de que se embarcara en un viaje a Italia en estas mismas fechas y una enfermedad que le había tenido indispuesto en esos mismos momentos, como se menciona en la correspondencia que Friedrich Münzer le envió desde Münster el 15 de julio de 1939, en respuesta a una anterior del propio Syme (Vivas García, 2016: 185, 244-245). He aquí un extracto de la misma: 
“Münster (Westf.) d. 15.7.39

Heisstr. 1.

Sehr geehrter Herr Kollege!

Nachdem unser Austausch von Briefen und Druckbogen im Anfang des Jahres so lebhaft gewesen war, habe ich mich allerdings gewundert und es mir nicht erklären können, warum er plötzlich vollkommen abgebrochen war. Mehr als einmal habe ich seitdem überlegt, ob ich an Sie schreiben sollte; aber ich wollte nicht aufdringlich erscheinen. Nun bin ich erfreut und dankbar, dass Ihr liebenswürdiger Brief mir die Aufklärung und eine Art von Beruhigung gibt; ich hoffe, dass Sie von Ihrer italienischen Reise befriedigt und von Ihrem Unwohlsein wiederhergestellt sind...."

Vemos que Münzer se había preguntado por qué Syme no le había escrito desde comienzos de año. Y que ahora se congratula de haber recibido respuesta de Syme en la que le explica las razones, a la vez que espera que haya disfrutado de su viaje a Italia y que esté recuperado de su indisposición, "Ihrem Unwohlsein".

Es posible que la jornada italiana y la dolencia sean la causa del retraso de esa quincena de días a los que el neozelandés se refiere en la carta de 14 de junio que estamos analizando. Quizá alguien de la editorial, que no fuera Sisam, hiciera notar a Syme esta tardanza y de ahí la respuesta de éste en el sentido de "not/ conscious of having been / a cause of delay in / the last fortnight". En uno u otro caso, estamos ante una carta de trascendencia puesto que atañe directamente a la fecha de publicación de su obra más conocida y admirada.

La última línea de la carta de Syme es también interesante, puesto que da a entender que, una vez publicado su libro, la editorial universitaria podía contar siempre con él como asesor para que emitiera sus opiniones acerca de las bondades o no de los distintos manuscritos que pudieran llegar a la Clarendon Press sobre temas de su especialidad. De hecho, Syme fue durante muchos años un colaborador muy activo de la editorial.

Con este trabajo hemos querido dar a conocer dos documentos inéditos hasta el momento y, en nuestra opinión, de importancia para saber un poco más del proceso de gestación de The Roman Revolution. 


\section{Bibliografía}

BOWERSOCK, Glen: “Ronald Syme 1903-1989”, Proceedings of the British Academy 84, 1994, p. 539-563.

STRAY, Christopher: “Kenneth Sisam (1887-1971)”, Oxford Dictionary of National Biography, https://doi.org/10.1093/ref:odnb/94507, publicado online: 26 de mayo de 2016.

SYME, Ronald: The Roman Revolution, Oxford: Clarendon Press, 1939.

VIVAS GARCÍA, Gustavo: Ronald Syme. El camino hasta La Revolución Romana (1928-1939), Barcelona: Universidad de Barcelona (Col ecciò Instrumenta 54), 2016.

. "El Ottaviano Capoparte de Mario Atilio Levi y su influencia en The Roman Revolution de Ronald Syme", Gerión 35 (1), 2017, p. 277-295.

. “La introducción del manuscrito de The Roman Revolution (Ms. Eng.

D. 2099): algunos aspectos", 2018, en prensa. 